\title{
Flow patterns behind the free flow front for a Newtonian fluid injected between two infinite parallel plates
}

\section{Citation for published version (APA):}

Gramberg, H. J. J., Vroonhoven, van, J. C. W., \& Ven, van de, A. A. F. (2003). Flow patterns behind the free flow front for a Newtonian fluid injected between two infinite parallel plates. (RANA : reports on applied and numerical analysis; Vol. 0305). Technische Universiteit Eindhoven.

Document status and date:

Published: 01/01/2003

\section{Document Version:}

Publisher's PDF, also known as Version of Record (includes final page, issue and volume numbers)

\section{Please check the document version of this publication:}

- A submitted manuscript is the version of the article upon submission and before peer-review. There can be important differences between the submitted version and the official published version of record. People interested in the research are advised to contact the author for the final version of the publication, or visit the $\mathrm{DOI}$ to the publisher's website.

- The final author version and the galley proof are versions of the publication after peer review.

- The final published version features the final layout of the paper including the volume, issue and page numbers.

Link to publication

\section{General rights}

Copyright and moral rights for the publications made accessible in the public portal are retained by the authors and/or other copyright owners and it is a condition of accessing publications that users recognise and abide by the legal requirements associated with these rights.

- Users may download and print one copy of any publication from the public portal for the purpose of private study or research.

- You may not further distribute the material or use it for any profit-making activity or commercial gain

- You may freely distribute the URL identifying the publication in the public portal.

If the publication is distributed under the terms of Article 25fa of the Dutch Copyright Act, indicated by the "Taverne" license above, please follow below link for the End User Agreement:

www.tue.nl/taverne

Take down policy

If you believe that this document breaches copyright please contact us at:

openaccess@tue.nl

providing details and we will investigate your claim. 


\title{
Flow patterns behind the free flow front for a Newtonian fluid injected between two infinite parallel plates.
}

\author{
H.J.J. Gramberg ${ }^{\mathrm{a}}$, J.C.W. van Vroonhoven ${ }^{\mathrm{b}}$ and \\ A.A.F. van de Ven ${ }^{a}$ \\ ${ }^{a}$ Mathematics and Computing Science, Technische Universiteit Eindhoven, \\ 5600MB Eindhoven, The Netherlands \\ ${ }^{\mathrm{b}}$ LG. Philips Displays Netherlands B.V., 5600 AV Eindhoven, The Netherlands
}

\begin{abstract}
A complete analytical treatment of the 2-dimensional problem of the injection of a Newtonian fluid between two parallel plates is presented. Explicit formulas are derived for the shape of the free flow front, the streamlines behind the flow front, the velocity, deformation and rotation (orientation) of material elements in the flow front region, and the associated stresses there. The analysis is based on complex function theory, and in this, the flow region, inclusive the unknown free flow front, is mapped onto the interior of the unit circle. The mapping function that determines the shape of the flow front is found by solving a Hilbert problem. It is analytically found in how far the actual flow front differs from a semi-circular shape, and it is concluded that the semi-circular approximation seems acceptable. Deformations of material line or area elements due to the fountain flow in the flow front region are followed in time; large deformation and reorientations of the material elements are observed. Our results are compared with results in literature obtained by numerical simulations and by experimental work, and on the whole good correspondence is found.
\end{abstract}

Keywords: injection moulding; free flow front; fountain flow; molecular orientation; conformal mapping.

\section{Introduction}

Injection moulding, especially in the filling phase, is a process exhibiting several peculiar but interesting aspects. To name some: the flow has a free boundary, the flow front, behind which a fountain flow occurs. Induced by this fountain flow, changes of molecular orientation in the injected polymer take place. 
Questions arise such as: what is the shape of the free flow front, how are the streamlines of the fountain flow directed, and how does the flow deform and reorientate a material line element moving to the wall of the mould? More advanced questions are: is the shape of the flow front stationary or does it change ("wobble"), and is this flow always stable? In this paper, we will restrict ourselves to injection moulding between two parallel plates; in this case the mould consists of a narrow striplike (2-dimensional) cavity bounded by two fixed walls.

Many articles have dealt with these subjects. The shape of the flow front was already calculated in the 80ties by, [1], [2], and [3]. Here, Diereck, [1], and Mavridis et al, [2], used finite element computations to calculate the front shape, whereas Vroonhoven and Kuijpers, [3], introduced a completely analytical approach, leading to an explicit formula for the flow front. Recent papers show more advanced numerical techniques; e.g. Friedrichs and Güçeri, [4], applied an hybrid 2-D/3-D numerical technique to compute the flow front, and the fountain flow behind it, for a shear-thinning non-Newtonian fluid. Such a 2-D/3-D approach was also proposed by Almeida et al, [5], by means of a so called Dimensional Reduction Method, based on a minimum energy principle. Other authors, such as Nguyen-Chung and Mennig, [6], used front tracking methods, whereas Pichelin and Coupez, [7], used a global transport equation to find the flow front position. In [7, Figs. 6 and 7], flow fronts for a Newtonian and a non-Newtonian, shear-thinning, fluid are compared; no great qualitative differences are found. A visualisation of the flow front behaviour by means of a flow front tracking camera system was recently presented by Yokoi et al, [8]. Results for the streamlines of the fountain flow behind the flow front are incorporated in e.g. [1], [2], [10], and [9]. Mavridis et al, [9], exploiting a Leonov viscoelastic model, also pictured the deformation of a material fluid element behind the flow front; when comparing with earlier results, [2], for a Newtonian fluid, the authors concluded that similar deformation patterns were found. In [6], an interesting figure, depicting the deformation of an initially straight, transverse, material line, can be found.

A paper deserving special interest is the one from Kamal et al, [11], who performed a numerical simulation for a polymer melt. They computed velocity profiles and stresses for two constitutive models: a power-law fluid and a viscoelastic (White-Metzner) model. Comparing the results, they found essentially the same velocity profiles (with only minor quantitative differences in the transverse profile), but significant differences in the stress profiles. The latter did not so much hold for the shear stresses inside the flow front region (see [11, Fig. 7, CP- and (CP+2)-lines]), but the more for the first normal stress difference ([11, Fig. 8]). The authors also replace, in order to alleviate the singularities occurring at the separation point, the no-slip condition at the walls by a slip condition in a small region behind the separation point. This modification affects the behaviour near the walls in the vicinity of the separation point essentially; this shows up most evident in the shear stresses (see [11, Fig. 5]). 
Molecular orientation, especially with regard to aspects such as residual stresses, birefringence, surface defects, and flow front instabilities, was studied in a series of references, of which we mention here [6], [9], [12], [13], [14], and [15]. In his thesis, Wimberger-Friedl, [13], mainly focussed on the effects of the molecular orientation on birefringence. Hung and Shen, [14], calculated the fibre orientation using Jeffery's model for a generalised Newtonian fluid. Vincent et al, [15], looked at the orientation of short fibres in reinforced thermoplastics due to fountain flow. Effects on surface defects and on flow front instabilities are thoroughly investigated by Grillet et al, [16], and by Bogaerds et al, [17], [18], respectively.

Except in [3], all the results in the papers listed above are derived by numerical means. For our purposes, we prefer an analytical approach, as in [3], and we want to calculate the shape of the flow front, the velocity field behind the flow front, the induced deformation and reorientation of material line elements, and the resulting stresses, all by purely analytical means. In doing this, we shall follow the lines set up by Vroonhoven and Kuijpers, [3]; in particular, in Section 3, we shall shortly recapitulate how they came to their explicit formula for the shape of the flow front. Starting from this result, we then proceed with the calculations of velocities, deformations, and stresses. Of course, these purely analytical calculations can only be done for a simple case. Therefore, we restrict ourselves to a two-dimensional injection between two parallel plates of an incompressible Newtonian fluid, with a no-slip boundary condition at the walls of the mould. Thermal effects are not taken into account. The shape of the flow front is fixed and moving with constant velocity. Far behind the flow front, the flow is a Poiseuille flow. The analysis is based on the theory of complex functions, [19], and on a conformal mapping of the flow front onto a unit circle. The specific form of this conformal mapping is the principal unknown in this problem. Once the mapping is known, all other quantities can be calculated. It turns out that the shape of the flow front is very close to, but not identically equal to, a semi-circle, as is conformed by several other articles, [1], [2] (see also Table 1), [10], [11] and [17](see Fig. 5.9).

In Section 4, the results of Section 3 are further evaluated to explicit formulas for especially the velocity in the flow front region. Other kinematical quantities such as deformations and rotations, as well as dynamical ones as sttresses, can then be analytically calculated. The results are presented in Section 5, while the main conclusions are listed in Section 6. As far as the kinematical results concern, the behaviour as found here corresponds very well with behaviour found in literature for much more complex fluid models. Therefore, a Newtonian model seems adequate enough to describe in a first order the kinematics of fountain flow for polymer melts of general constitutive nature (Non-Newtonian, nonlinear viscoelastic). 


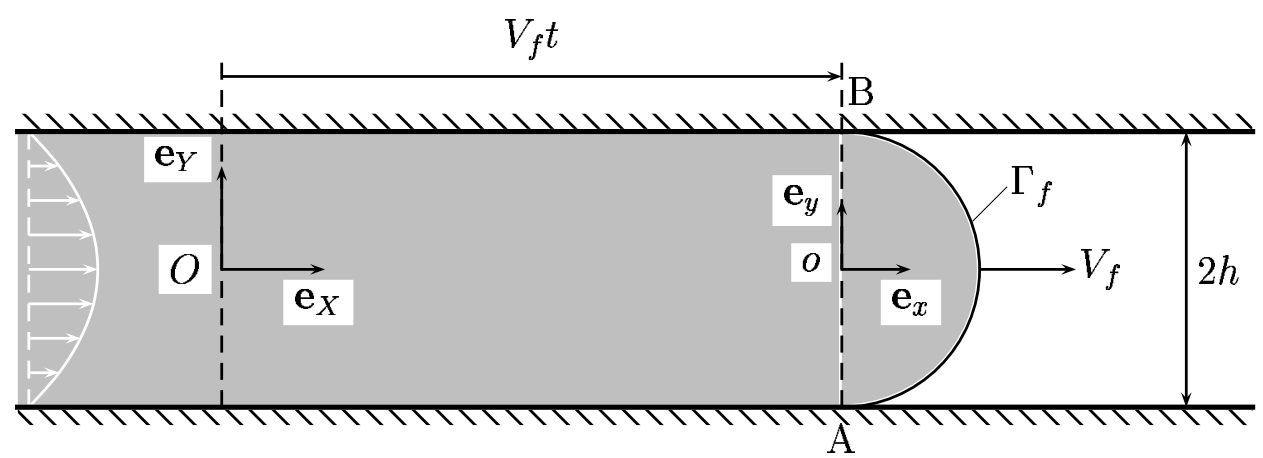

Fig. 1. The flow region with moving front between two plates.

\section{Problem description}

A fluid is injected with prescribed volumetric flow rate $Q$ between two infinite parallel plates at distance $2 h$. The flow is 2-dimensional in the $X-Y$-plane; see Figure 1. The fixed origin $O$ of an $\{O X Y\}$ system is midway between the plates, the $X$-axis points in the flow direction and the $Y$-axis is perpendicular to the plates $(-h<Y<h)$. In the flow considered here, the flow front $\Gamma_{f}$ is an unknown free surface. In the next section, we shall show how the shape of $\Gamma_{f}$ can be determined completely by analytical means. For this, we restrict ourselves to an incompressible Newtonian fluid model, in which case a fully analytical solution can be obtained.

We assume the flow so slow, and the viscosity of the fluid so high, that all inertia effects may be neglected (small Reynolds number $\Rightarrow$ Stokes flow). Moreover, the shape of the flow front is taken constant (stationary) and the front moves with constant speed $V_{f}$. This speed is related to the given flow rate $Q$, per unit of length in the direction normal to the plane of flow, by

$$
V_{f}=\frac{Q}{2 h} .
$$

Far behind the flow front, the flow is a fully developed Poiseuille flow. Here, the velocity $\mathbf{V}=U \mathbf{e}_{X}+V \mathbf{e}_{Y}$ is given by

$$
U=\frac{3 Q}{4 h}\left[1-\left(\frac{Y}{h}\right)^{2}\right]=\frac{3}{2} V_{f}\left[1-\left(\frac{Y}{h}\right)^{2}\right], \quad V=0 .
$$

The actual flow front region is given by the area between the line $A B$, see Figure 1, and the free flow front $\Gamma_{f}$. Note that this area moves with constant speed $V_{f}$. Therefore, we introduce a comoving frame $\{o x y\}$, as depicted in 
Figure 1, with the dimensionless coordinates

$$
x=\frac{X-V_{f} t}{h}, \quad y=\frac{Y}{h}, \quad(-1<y<1) .
$$

In this comoving frame, the flow front is given by

$$
\Gamma_{f}(x, y)=0
$$

where the function $\Gamma_{f}(x, y)$ is still to be determined.

The dimensionless velocity $\mathbf{v}=u(x, y) \mathbf{e}_{x}+v(x, y) \mathbf{e}_{y}$ with respect to the comoving frame is related to the dimensional absolute velocity $\mathbf{V}(X, Y, t)$ by

$$
U=V_{f}(1+u), \quad V=V_{f} v
$$

The incompressibility condition $\operatorname{div} \mathbf{v}=0$, written here as

$$
\frac{\partial u}{\partial x}+\frac{\partial v}{\partial y}=0
$$

allows us to introduce a stream function $\psi(x, y)$ by

$$
u=\frac{\partial \psi}{\partial y}, \quad v=-\frac{\partial \psi}{\partial x}
$$

For a Newtonian fluid model, the stress tensor $\mathcal{T}$ is given by the constitutive equation

$$
\mathcal{T}=-p \mathcal{I}+2 \eta \mathcal{D}
$$

where $p=p(x, y)$ is the pressure, $\eta$ is the (constant) viscosity and $\mathcal{D}$ is the rate of deformation tensor. Scaling the pressure and the stress with a factor $h / 2 \eta V_{f}$, we obtain the dimensionless formulation (since confusion is unlikely, we attain the same notation for $p$ and $\mathcal{T}$ )

$$
\mathcal{T}=-p \mathcal{I}+\mathcal{D}
$$

where

$$
\mathcal{D}=\frac{1}{2}\left(\nabla \mathbf{v}+(\nabla \mathbf{v})^{T}\right)
$$

In the absence of body forces and under the neglect of inertia terms, the equation of motion reduces to

$$
\operatorname{div} \mathcal{T}=\mathbf{0}
$$

This equation can be identically satisfied by introducing an Airy stress function $\phi(x, y)$ such that

$$
T_{x x}=-\frac{\partial^{2} \phi}{\partial y^{2}}, \quad T_{x y}=\frac{\partial^{2} \phi}{\partial x \partial y}, \quad T_{y y}=-\frac{\partial^{2} \phi}{\partial x^{2}},
$$


while, then

$$
p(x, y)=\frac{1}{2} \triangle \phi=\frac{1}{2}\left(\frac{\partial^{2} \phi}{\partial x^{2}}+\frac{\partial^{2} \phi}{\partial y^{2}}\right) .
$$

Use of the constitutive equation (9) shows that $\psi$ and $\phi$ are related to each other by

$$
\frac{\partial^{2} \psi}{\partial x \partial y}=\frac{1}{2}\left(\frac{\partial^{2} \phi}{\partial x^{2}}-\frac{\partial^{2} \phi}{\partial y^{2}}\right), \quad \frac{\partial^{2} \phi}{\partial x \partial y}=-\frac{1}{2}\left(\frac{\partial^{2} \psi}{\partial x^{2}}-\frac{\partial^{2} \psi}{\partial y^{2}}\right)
$$

These relations imply that both $\psi$ and $\phi$ have to satisfy a biharmonic equation.

For the boundary conditions, we have

- at the walls $y= \pm 1$ : a no-slip condition, i.e. $\mathbf{V}=\mathbf{0}$, yielding

$$
u(x, \pm 1)=-1, \quad v(x, \pm 1)=0 ;
$$

- for $x \rightarrow-\infty$ : fully developed Poiseuille flow, according to (2), yielding

$$
u(x, y) \rightarrow u_{0}(y)=\frac{1}{2}-\frac{3}{2} y^{2}, \quad v(x, y) \rightarrow 0
$$

- at the flow front $(x, y) \in \Gamma_{f}$, being a free flow front, we require

$$
\mathcal{T} \mathbf{n}=-p_{0} \mathbf{n}
$$

where $\mathbf{n}$ is the unit outward normal vector on $\Gamma_{f}$, and $p_{0}$ is the environmental pressure.

Finally, we need the free-flow-front condition, stating that the front curve $\Gamma_{f}$ must be a flow line. This is expressed by

$$
(\mathbf{v}, \mathbf{n})=0, \quad \text { for }(x, y) \in \Gamma_{f} .
$$

\section{Complex formulation and solution}

In this section, we only present the main lines for the determination of $\Gamma_{f}$, by means of the theory of complex functions and conformal mapping. For the details of the derivations, we refer to [3].

\subsection{Complex potentials}

Upon introducing the complex variables

$$
z=x+\mathrm{i} y, \quad \bar{z}=x-\mathrm{i} y
$$


we can express the complex function

$$
\Phi=\phi(x, y)+\mathrm{i} \psi(x, y)
$$

in terms of $z$ and $\bar{z}$, i.e. $\Phi=\Phi(z, \bar{z})$. This function is governed by the equation (following from (14))

$$
\frac{\partial^{2} \Phi(z, \bar{z})}{\partial \bar{z}^{2}}=0
$$

implying that $\Phi(z, \bar{z})$ can be expressed in terms of the analytical functions $\Omega(z)$ and $\omega(z)$ as

$$
\Phi(z, \bar{z})=\bar{z} \Omega(z)+\omega(z) .
$$

We can now also express the complex velocity $w=u+\mathrm{i} v$ and the stresses in terms of $\Omega(z)$ and $\omega(z)$, resulting in (' means differentiation with respect to $z$, and $\overline{f(z)}$ denotes the complex conjugate of $f(z))$

$$
\begin{aligned}
w(z, \bar{z}) & =z \overline{\Omega^{\prime}(z)}+\overline{\omega^{\prime}(z)}-\Omega(z) \\
N(z, \bar{z}) & =T_{x x}+T_{y y}=-2\left(\Omega^{\prime}(z)+\overline{\Omega^{\prime}(z)}\right), \\
S(z, \bar{z}) & =T_{x x}-T_{y y}+2 \mathrm{i} T_{x y}=2 z \overline{\Omega^{\prime \prime}(z)}+2 \overline{\omega^{\prime \prime}(z)} .
\end{aligned}
$$

To eliminate the influence of the environmental pressure $p_{0}$ and of the flow far behind the flow front $\left(w \rightarrow u_{0}(y)\right.$ for $\left.\operatorname{Re}(z) \rightarrow-\infty\right)$, we write $\Omega(z)=$ $\Omega_{0}(z)+\Omega_{1}(z)$ and $\omega(z)=\omega_{0}(z)+\omega_{1}(z)$, where

$$
\Omega_{0}(z)=-\frac{1}{4}\left(1+\frac{3}{2} z^{2}\right)+\frac{1}{2} p_{0} z, \quad \omega_{0}(z)=\frac{1}{4} z\left(1+\frac{1}{2} z^{2}\right) .
$$

With this modification, (23) becomes (with $w_{1}=w-w_{0}, w_{0}(z, \bar{z})=u_{0}(y)$ )

$$
\begin{aligned}
& w_{1}(z, \bar{z})=z \overline{\Omega_{1}^{\prime}(z)}+\overline{\omega_{1}^{\prime}(z)}-\Omega_{1}(z) \\
& N(z, \bar{z})=T_{x x}+T_{y y}=-2\left(\Omega_{1}^{\prime}(z)+\overline{\Omega_{1}^{\prime}(z)}\right)+\frac{3}{2}(z+\bar{z})-2 p_{0} \\
& S(z, \bar{z})=T_{x x}-T_{y y}+2 \mathrm{i} T_{x y}=2 z \overline{\Omega_{1}^{\prime \prime}(z)}+2 \overline{\omega_{1}^{\prime \prime}(z)}-\frac{3}{2}(z-\bar{z})
\end{aligned}
$$

We can now express the boundary conditions (15)-(18) in terms of $\Omega_{1}(z)$ and $\omega_{1}(z)$. This yields successively

$$
\begin{aligned}
z \overline{\Omega_{1}^{\prime}(z)}+\overline{\omega_{1}^{\prime}(z)}-\Omega_{1}(z) & =0, \quad \operatorname{Im}(z)= \pm 1, \\
z \overline{\Omega_{1}^{\prime}(z)}+\overline{\omega_{1}^{\prime}(z)}-\Omega_{1}(z) & \rightarrow 0, \quad \operatorname{Re}(z) \rightarrow-\infty, \\
z \overline{\Omega_{1}^{\prime}(z)}+\overline{\omega_{1}^{\prime}(z)}+\Omega_{1}(z) & =\frac{3}{8}\left(z^{2}+2 z \bar{z}-\bar{z}^{2}\right), \quad z \in \Gamma_{f}, \\
\operatorname{Re}\left[\left(z \overline{\Omega_{1}^{\prime}(z)}+\overline{\omega_{1}^{\prime}(z)}-\Omega_{1}(z)\right)\left(n_{x}-\mathrm{i} n_{y}\right)\right] & =\frac{3}{2}(\operatorname{Im}(z))^{2}-\frac{1}{2}, \quad z \in \Gamma_{f} \cdot(26)
\end{aligned}
$$




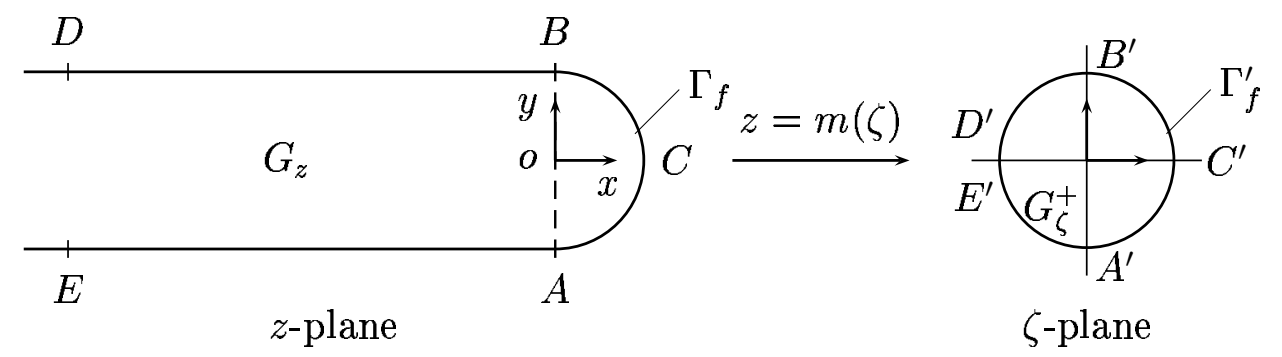

Fig. 2. The conformal mapping of $G_{z}$ onto the unit circle $G_{\zeta}^{+}$.

We note here that the third of the conditions above does not prescribe the stresses, as in (17), but rather the resultant force on a part of the $\operatorname{arc} \Gamma_{f}$. This is conform to [3] and [19, Eq. (2.47)].

\subsection{Conformal mapping}

The basic idea of the method used in [3] is to map the total flow region $G_{z}$ in the $z$-plane onto the unit circle $G_{\zeta}^{+}$in the $\zeta$-plane and then to reduce the problem to a so called Hilbert problem on the unit circle, that can explicitly be solved in terms of the conformal mapping $m(\zeta)$. In this way, the problem of determining the explicit shape of the flow front is tackled completely by analytical means. The conformal mapping $z=m(\zeta)$, depicted in Figure 2, is by definition analytic, implying that it can be expressed as an infinite power series, with radius of convergence 1 ,

$$
m(\zeta)=\sum_{k=0}^{\infty} \mu_{k} \zeta^{k}
$$

which will be approximated by the truncated series

$$
m_{N}(\zeta)=\sum_{k=0}^{N} \mu_{k} \zeta^{k}
$$

Note that, as the shape of $\Gamma_{f}$ is unknown, the real coefficients $\mu_{k}$ in (28) are still unknown.

We assume that $m_{N}(\zeta)$ is a good approximation of the mapping function $m(\zeta)$, especially in the neighbourhood of the free boundary (this will be confirmed by the final results). Since $m_{N}(\zeta)$ is a polynomial of degree $N$, it is clearly analytic and a one-to-one mapping as long as $N$ is not too large.

Next, we replace $\Omega_{1}(z)$ and $\omega_{1}(z)$ by functions of $\zeta$ by means of the substitution $z=m_{N}(\zeta)$, yielding

$$
\Omega_{N}(\zeta)=\Omega_{1}\left(m_{N}(\zeta)\right), \quad \omega_{N}(\zeta)=\omega_{1}\left(m_{N}(\zeta)\right)
$$


For the complex velocity $w_{1}(z, \bar{z})$, given by $(25.1)$, this for instance yields

$$
w_{N 1}=\frac{m_{N}(\zeta) \overline{\Omega_{N}^{\prime}(\zeta)}+\overline{\omega_{N}^{\prime}(\zeta)}}{\overline{m_{N}^{\prime}(\zeta)}}-\Omega_{N}(\zeta)
$$

We proceed with the evaluation of the boundary conditions. In the following, a superscript ${ }^{+}$denotes the boundary value of the function coming from the interior of the unit circle, so e.g.

$$
\Omega_{N}^{+}(\xi)=\lim _{\zeta \rightarrow \xi, \zeta \in G_{\zeta}^{+}} \Omega_{N}(\zeta), \quad \xi \in \Gamma_{f}^{\prime}
$$

Thus, we obtain from the first two boundary conditions of (26)

$$
\frac{m_{N}^{+}(\xi) \overline{\Omega_{N}^{\prime+}(\xi)}+\overline{\omega_{N}^{\prime+}(\xi)}}{\overline{m_{N}^{\prime+}(\xi)}}-\Omega_{N}^{+}(\xi)=0, \quad \xi \in A^{\prime} E^{\prime} D^{\prime} B^{\prime}
$$

and for the third one

$$
\frac{m_{N}^{+}(\xi) \overline{\Omega_{N}^{\prime+}(\xi)}+\overline{\omega_{N}^{+\prime}(\xi)}}{\overline{m_{N}^{\prime+}(\xi)}}+\Omega_{N}^{+}(\xi)=g_{N}(\xi), \quad \xi \in \Gamma_{f}^{\prime}
$$

where

$$
g_{N}(\xi)=\frac{3}{8}\left(m_{N}(\xi)^{2}+2 m_{N}(\xi) \overline{m_{N}(\xi)}-\overline{m_{N}^{2}(\xi)}\right)
$$

For the evaluation of the fourth boundary condition of (26), we need the analytical continuation of $\Omega_{N}(\zeta)$ to the exterior space $G_{\zeta}^{-}$of the unit circle. This is done by introducing the function $\Psi_{N}(\zeta)$ in the following way

$$
\begin{array}{ll}
\Psi_{N}(\zeta)= & \Omega_{N}(\zeta), \\
\Psi_{N}(\zeta)=\frac{m_{N}(\zeta) \overline{\Omega_{N}^{\prime}(1 / \bar{\zeta})}+\overline{\omega_{N}^{\prime}(1 / \bar{\zeta})}}{\overline{m_{N}^{\prime}(1 / \bar{\zeta})}}, \zeta \in G_{\zeta}^{+},
\end{array}
$$

We note that $\Omega_{N}(\zeta)$ is, by definition, continuous over the $\operatorname{arc} A^{\prime} E^{\prime} D^{\prime} B^{\prime}$ (in accordance with (32)) and, hence, analytic for $\zeta \in \mathbb{C} \backslash \Gamma_{f}^{\prime}$. With (35), the function $\omega_{N}$ can be expressed in $\Psi_{N}$ and $m_{N}$ according to

$$
\omega_{N}^{\prime}(\zeta)=m_{N}^{\prime}(\zeta) \overline{\Psi_{N}(1 / \bar{\zeta})}-\overline{m_{N}(1 / \bar{\zeta})} \Psi_{N}^{\prime}(\zeta), \quad \zeta \in G_{\zeta}^{+}
$$

Both the functions $\overline{m_{N}(1 / \bar{\zeta})}$ and $\overline{\Psi_{N}(1 / \bar{\zeta})}$ have poles of order $N$ at the origin. The analycity of $\omega$, however, demands that these poles cancel at the right-hand side of (36). This will be referred to as the holomorphy condition. Moreover, the function $\Psi_{N}$ has to satisfy some regularity conditions, such as

$$
\Psi_{N}(\zeta)=\mathcal{O}(1), \quad \zeta \rightarrow \pm \mathrm{i}
$$


expressing that the velocity must remain finite near the points $A$ and $B$, and

$$
\Psi_{N}(\zeta)=\mathcal{O}\left(\zeta^{N}\right), \quad|\zeta| \rightarrow \infty
$$

which follows immediately from the definition of $\Psi_{N}$.

All this leads us to the formulation of the following Hilbert problem for $\Psi_{N}$ (here, $\Psi_{N}^{-}(\xi)=\lim _{\zeta \rightarrow \xi, \zeta \in G_{\zeta}^{-}} \Psi_{N}(\zeta)$ )

$$
\begin{aligned}
& \Psi_{N}^{+}(\xi)-\Psi_{N}^{-}(\xi)=0, \quad \xi \in A^{\prime} E^{\prime} D^{\prime} B^{\prime}, \\
& \Psi_{N}^{+}(\xi)+\Psi_{N}^{-}(\xi)=g_{N}(\xi), \quad \xi \in \Gamma_{f}^{\prime} \text {. }
\end{aligned}
$$

The solution of this Hilbert problem will be presented in the next section.

Finally, for the determination of $m_{N}(\zeta)$ we need the flow-front condition $(\mathbf{v}, \mathbf{n})=0$ on $\Gamma_{f}^{\prime}$, as expressed by the fourth boundary condition of (26), which can be evaluated into

$$
\operatorname{Re}\left(\left[\frac{1}{2}-\frac{3}{2}(\operatorname{Im} m(\xi))^{2}+g_{N}(\xi)-2 \Psi_{N}^{+}(\xi)\right] \overline{\xi m_{N}^{\prime}(\xi)}\right)=0, \quad \xi \in \Gamma_{f}^{\prime}
$$

Here, we have used (39) to eliminate $\Psi_{N}^{-}$, together with the relation

$$
n_{x}+\mathrm{i} n_{y}=-\mathrm{i} \frac{d z}{d s}=-\mathrm{i} m_{N}^{\prime+}(\xi) \frac{d \xi}{d s}=\xi m_{N}^{\prime+}(\xi)
$$

holding along the unit circle where $\frac{d \xi}{d s}=\mathrm{i} \xi$.

\subsection{Solution of the Hilbert problem}

The solution procedure of (39) is described in detail in [3], therefore, we here present the main lines only. The solution can be split up into a particular solution and a homogeneous one, looking like

$$
\Psi_{N}(\zeta)=X(\zeta) G_{N}(\zeta)+X(\zeta) F_{N}(\zeta)
$$

where $X(\zeta)$ is the Plemelj function:

$$
X(\zeta)=(\zeta-\mathrm{i})^{1 / 2}(\zeta+\mathrm{i})^{1 / 2}, \quad \zeta \in \mathbb{C} \backslash \Gamma_{f}^{\prime}
$$

satisfying

$$
X^{+}(\xi)+X^{-}(\xi)=0, \quad \xi \in \Gamma_{f}^{\prime}
$$

while $G_{N}$ is defined by the Cauchy integral,

$$
G_{N}(\zeta)=\frac{1}{2 \pi \mathrm{i}} \int_{\Gamma_{f}^{\prime}} \frac{g_{N}(\xi)}{X^{+}(\xi)(\xi-\zeta)} \mathrm{d} \xi
$$


and $F_{N}$ is a polynomial of degree $N-1$,

$$
F_{N}(\zeta)=\sum_{k=0}^{N-1} f_{k} \zeta^{k}
$$

the coefficients of which can be determined by means of the holomorphy condition.

The Plemelj function $X(\zeta)$ has branch points at $\zeta= \pm \mathrm{i}$, and the cut, joining -i with infinity is defined in such a way that; see [19],

$$
X(\zeta)=-\sqrt{\left(1+\zeta^{2}\right)} \quad \zeta \in G_{\zeta}^{+}, \quad X(\zeta)=\sqrt{\left(1+\zeta^{2}\right)} \quad \zeta \in G_{\zeta}^{-}
$$

Note that in (41) the first term $X(\zeta) G_{N}(\zeta)$ represents the particular solution, whereas the second term $X(\zeta) F_{N}(\zeta)$ stands for the homogeneous solution.

As will be shown in Section 4.1, the function $G_{N}(\zeta)$ can be explicitly expressed in the coefficients of the conformal mapping $m_{N}(\zeta)$. To this end, we consider the function $g_{N}(\xi)$ in the integrand of (44), defined in (34) for $\xi \in \Gamma_{f}^{\prime}$, as the limiting value of an analytical function $g_{N}(\zeta)$ (analytic in $\mathbb{C} \backslash \Gamma_{f}^{\prime}$ ) for $\zeta \rightarrow \xi \in \Gamma_{f}^{\prime}$. This function is given by

$$
g_{N}(\zeta)=\frac{3}{8}\left(m_{N}^{2}(\zeta)+2 m_{N}(\zeta) \overline{m_{N}(1 / \bar{\zeta})}-\overline{m_{N}^{2}(1 / \bar{\zeta})}\right)
$$

Then, the following expression for $G_{N}(\zeta)$ can be derived (this derivation is postponed to Section 4.1; see (65) for the result)

$$
2 G_{N}(\zeta)=\frac{g_{N}(\zeta)}{X(\zeta)}-\sum_{k=-2 N}^{-1} a_{k} \zeta^{k}-\sum_{k=0}^{2 N-1} b_{k} \zeta^{k}
$$

We introduce the Laurent series

$$
\begin{aligned}
& \frac{g_{N}(\zeta)}{X(\zeta)}=\sum_{k=-2 N}^{\infty} a_{k} \zeta^{k}, \quad \zeta \in G^{+}, \\
& \frac{g_{N}(\zeta)}{X(\zeta)}=\sum_{k=-\infty}^{2 N-1} b_{k} \zeta^{k}, \quad \zeta \in G^{-},
\end{aligned}
$$

the coefficients of which can be expressed in terms of $\mu_{k}$ (see (53) below). With the above results, the solution of the Hilbert problem can be written as

$$
\Psi_{N}(\zeta)=\frac{1}{2} g_{N}(\zeta)-\frac{1}{2} X(\zeta)\left(\sum_{k=-2 N}^{-1} a_{k} \zeta^{k}+\sum_{k=0}^{2 N-1} b_{k} \zeta^{k}-2 \sum_{k=0}^{N-1} f_{k} \zeta^{k}\right)
$$

for $\zeta \in \mathbb{C} \backslash \Gamma_{f}^{\prime}$. This means that, given the coefficients $\mu_{k}, k=0,1 \ldots N$, (by which also $g_{N}(\zeta)$ is given, according to (47)) the solution of the Hilbert problem is reduced to determining (by analytical means) only $5 N$ coefficients $a_{k}, b_{k}$ and $f_{k}$. What remains now is the calculation of $\mu_{k}, k=0,1 \ldots N$, from the 
free-boundary condition (40). The latter step can only be done numerically (however, by a very simple procedure). Results for several values of $N$ are presented by Vroonhoven and Kuijpers in [3]. As an example, for the case $N=3$ they found

$$
\mu_{0}=-0.04287, \quad \mu_{1}=0.98349, \quad \mu_{2}=-0.04287, \quad \mu_{3}=-0.01651
$$

In Figure 3, the exact flow front is compared with a semi-circle, and a reasonable resemblance is found; a similar result is found in [2, Figs. 13-14]. A typical set of flow lines in the flow region, with respect to the comoving frame, for the $\mu$-values according to (51) is depicted in Figure 5.b. We note that these flow lines are completely in agreement with similar results obtained in [2], [10], [9], and [17], all by finite element simulations, and often for more complex fluid models than the Newtonian model used here.

\section{Further evaluation of the results of Section 3}

\subsection{Calculation of complex velocity}

As mentioned in the previous section, if $N$ is given and $\mu_{k}$ for $k=0, \ldots, N$ are known, we can determine the coefficients $a_{k}, b_{k}$ and $f_{k}$ analytically. In this section, we will outline the procedure how to do this, and how this will eventually result in an explicit expression for the complex velocity $w_{N}(\zeta)$.

The coefficients $a_{k}$ and $b_{k}$ follow from their definitions, and can be calculated explicitly in terms of $\mu_{k}$. Since $\mu_{k} \in \mathbb{R}$, it holds that $\bar{\mu}_{k}=\mu_{k}$. Therefore,

$$
\overline{m_{N}(1 / \bar{\zeta})}=\sum_{i=0}^{N} \mu_{i} \zeta^{-i}, \quad \zeta \in \mathbb{C} \backslash\{0\}
$$

Moreover, for the analytical function $g_{N}(\zeta)$ introduced in (47), we have for $\zeta \in \mathbb{C} \backslash\{0\}$,

$$
g_{N}(\zeta)=\frac{3}{8}\left(\sum_{i=0}^{2 N} \sum_{j=0}^{N} \mu_{j} \mu_{i-j} \zeta^{i}+2 \sum_{i=-N}^{N} \sum_{j=0}^{N} \mu_{j} \mu_{j-i} \zeta^{i}-\sum_{i=-2 N}^{0} \sum_{j=0}^{N} \mu_{j} \mu_{-j-i} \zeta^{i}\right)
$$

where we define $\mu_{i}=0$ for $i<0$ or $i>N$. For $\zeta \rightarrow 0,1 / X(\zeta)$ can be approximated according to (see (46))

$$
\frac{1}{X(\zeta)}=-\left(1+\zeta^{2}\right)^{-1 / 2}=\frac{8}{3} \sum_{j=0}^{\infty} \lambda_{j} \zeta^{j}
$$




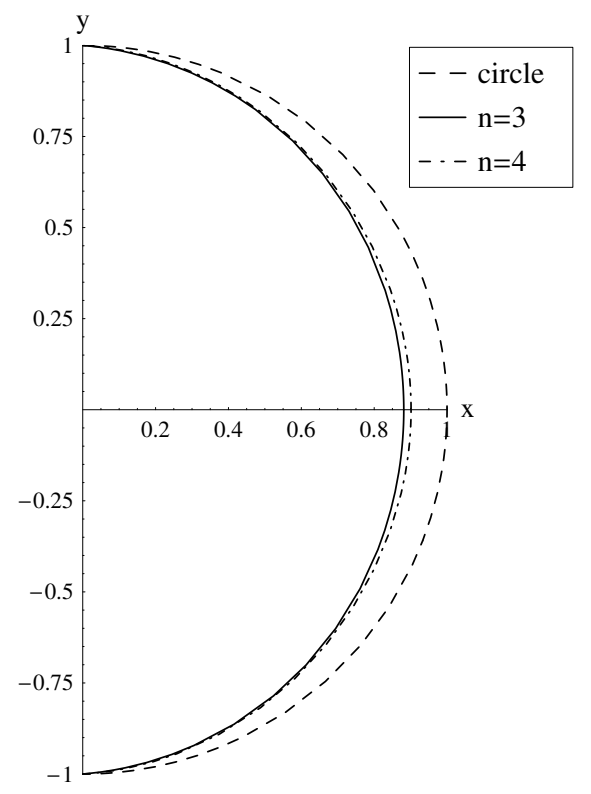

Fig. 3. The exact flow front compared with a semi-circle

with $\lambda_{j}$ equal to

$$
\lambda_{j}:= \begin{cases}-\frac{3}{8}\left(\begin{array}{c}
-1 / 2 \\
k
\end{array}\right), & \text { for } j=2 k, k \in \mathbb{N} \cup\{0\} \\
0, & \text { for } j \text { odd or } j<0,\end{cases}
$$

where $\left(\begin{array}{c}-1 / 2 \\ k\end{array}\right)$ is defined according to

$$
\left(\begin{array}{c}
-1 / 2 \\
k
\end{array}\right)=\frac{(-1)^{k}}{4^{k}} \frac{(2 k) !}{(k !)^{2}}, \quad k \in \mathbb{N} \cup\{0\}
$$

If we combine (53) and (55), to get an expression for $g_{N}(\zeta) / X(\zeta)$ in terms of $\zeta^{k}$, and if we use this in the definition of $a_{k},(49)$, it follows that $a_{k}$ is equal to

$$
a_{k}=\sum_{i=0}^{2 N} \lambda_{k-i} \sum_{j=0}^{N} \mu_{j} \mu_{i-j}+2 \sum_{i=-N}^{N} \lambda_{k-i} \sum_{j=0}^{N} \mu_{j} \mu_{j-i}-\sum_{i=-2 N}^{0} \lambda_{k-i} \sum_{j=0}^{N} \mu_{j} \mu_{-i-j},
$$

for $k \geq-2 N$. Analogously, we can develop $g_{N}(\zeta)$ and $X(\zeta)$ as Taylor series for $|\zeta| \rightarrow \infty$, rendering the following equation for $b_{k}$

$$
b_{k}=\sum_{i=-2 N}^{0} \gamma_{-k-i} \sum_{j=0}^{N} \mu_{j} \mu_{-i-j}+2 \sum_{i=-N}^{N} \gamma_{-k-i} \sum_{j=0}^{N} \mu_{j} \mu_{i+j}-\sum_{i=0}^{2 N} \gamma_{-k-i} \sum_{j=0}^{N} \mu_{j} \mu_{i-j}
$$



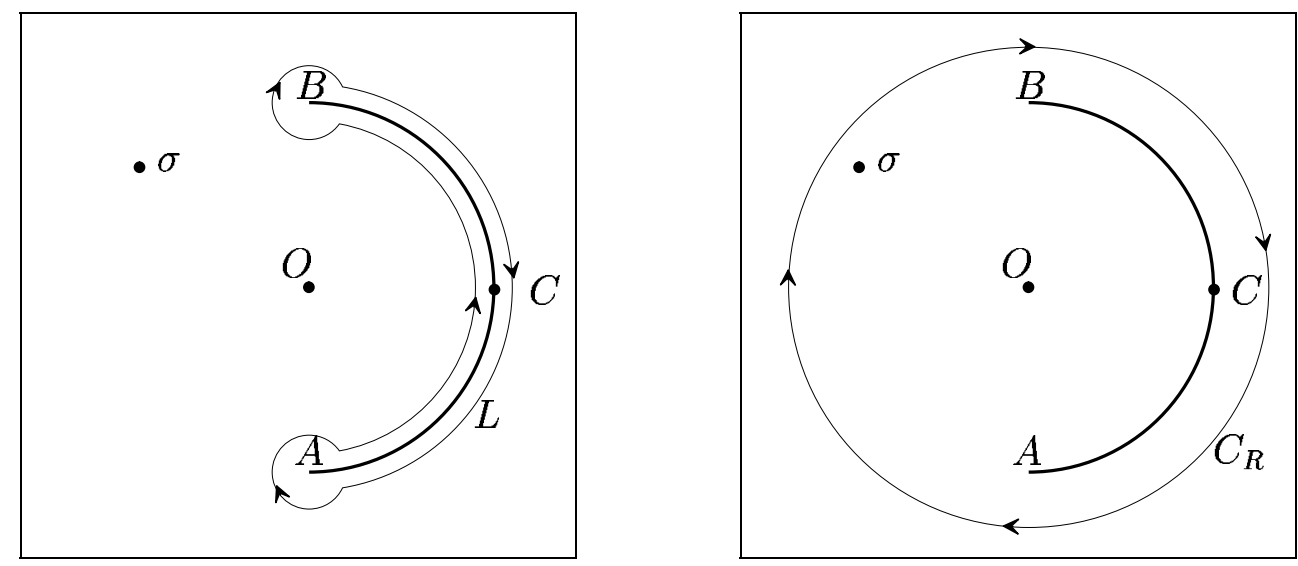

Fig. 4. Contours $L$ and $C_{R}$

where $k<2 N-1$, and $\gamma_{j}$ is given by

$$
\gamma_{j}:= \begin{cases}\frac{3}{8}\left(\begin{array}{c}
-1 / 2 \\
k
\end{array}\right), & \text { for } j=2 k+1, k \in \mathbb{N} \cup\{0\} \\
0, & \text { for } j \text { even or } j<0\end{cases}
$$

In order to calculate $f_{k}$, we first need to find an explicit expression for $G_{N}(\zeta)$ in terms of $a_{k}, b_{k}$ and $g_{N}$. Consider the following integrals

$$
\begin{aligned}
& I_{1}(\sigma):=\frac{1}{2 \pi i} \int_{L} \frac{g_{N}(\zeta)}{X(\zeta)(\zeta-\sigma)} \mathrm{d} \zeta \\
& I_{2}(\sigma):=\frac{1}{2 \pi i} \int_{C_{R}} \frac{g_{N}(\zeta)}{X(\zeta)(\zeta-\sigma)} \mathrm{d} \zeta
\end{aligned}
$$

where $L$ is a contour enclosing $\Gamma_{f}^{\prime}$, but such that the points 0 and $\sigma$ lie outside $L$, while $C_{R}$ is a circle with radius $R>\max \{1,|\sigma|\}$ as depicted in Figure 4 . By taking into account that

$$
\frac{g_{N}(\zeta)}{X(\zeta)(\zeta-\sigma)}=O\left(\delta^{-1 / 2}\right), \quad \text { for }|\zeta \pm \mathrm{i}|=: \delta \rightarrow 0
$$

and by using (43) and (44), we find the following expression for $I_{1}(\sigma)$

$$
I_{1}(\sigma)=\frac{1}{2 \pi \mathrm{i}} \int_{\Gamma_{f}^{\prime}} \frac{g_{N}(\xi)}{X^{+}(\xi)(\xi-\sigma)} \mathrm{d} \xi-\frac{1}{2 \pi \mathrm{i}} \int_{\Gamma_{f}^{\prime}} \frac{g_{N}(\xi)}{X^{-}(\xi)(\xi-\sigma)} \mathrm{d} \xi=2 G_{N}(\sigma)
$$

Since $g_{N}(\zeta) / X(\zeta)$ is holomorphic outside $C_{R}$ and $\sigma$ is a point inside $C_{R}$, the integral $I_{2}(\sigma)$ is equal to the residue of the integrand in infinity (see [19, eq.(1.15)]) and thus we find the following expression for $I_{2}(\sigma)$, with use of (49),

$$
I_{2}(\sigma)=-\sum_{k=0}^{2 N-1} b_{k} \sigma^{k}
$$


From the residue theorem of Cauchy, it follows that $I_{1}(\sigma)-I_{2}(\sigma)$ is equal to

$$
\begin{aligned}
I_{1}(\sigma)-I_{2}(\sigma) & =\operatorname{Res}_{\zeta=\sigma} \frac{g_{N}(\sigma)}{X(\zeta)(\zeta-\sigma)}+\operatorname{Res}_{\zeta=0} \frac{g_{N}(\sigma)}{X(\zeta)(\zeta-\sigma)}= \\
& =\frac{g_{N}(\sigma)}{X(\sigma)}-\sum_{k=-2 N}^{-1} a_{k} \sigma^{k}
\end{aligned}
$$

Combining (62)-(64), we find the following expression for $G_{N}(\zeta)$, valid in $\mathbb{C} \backslash\left\{\Gamma_{f}^{\prime}\right\}$,

$$
2 G_{N}(\zeta)=\frac{g_{N}(\zeta)}{X(\zeta)}-\sum_{k=-2 N}^{-1} a_{k} \zeta^{k}-\sum_{k=0}^{2 N-1} b_{k} \zeta^{k}
$$

With (65) we have found the explicit expression for $G_{N}(\zeta)$, we were looking for.

Using the first equation of (49), we see that the singularity in $\zeta=0$ is removable. For $|\zeta| \rightarrow \infty$, the second equation of (49) yields

$$
2 G_{N}(\zeta)=\sum_{k=-\infty}^{2 N-1} b_{k} \zeta^{k}-\sum_{-2 N}^{-1} a_{k} \zeta^{k}-\sum_{k=0}^{2 N-1} b_{k} \zeta^{k}=O(1 / \zeta)
$$

So, the function $G_{N}$ is holomorphic in $\mathbb{C} \backslash\left\{\Gamma_{f}^{\prime}\right\}$, including the point at infinity. We proceed with the calculation of the polynomial $F_{N}(\zeta)$ by considering the holomorphy condition, formulated in relation with (36). Consider the following equation, obtained by dividing (36) by $X(\zeta)$,

$$
-\frac{\omega_{N}^{\prime}(\zeta)}{X(\zeta)}=-\frac{m_{N}^{\prime}(\zeta) \overline{\Psi_{N}(1 / \bar{\zeta})}}{X(\zeta)}+\frac{\overline{m_{N}(1 / \bar{\zeta})} \Psi_{N}^{\prime}(\zeta)}{X(\zeta)}, \quad \zeta \in G_{\zeta}^{+}
$$

In order to satisfy the holomorphy condition, i.e. requiring that $\omega_{N}$ is analytical in $G_{\zeta}^{+}$, the right-hand side of (67) must remain finite for $\zeta \rightarrow 0$.

Since (see (46))

$$
\overline{X(1 / \bar{\zeta})}=-\frac{1}{\zeta} X(\zeta)
$$

we obtain from (41) with the aid of (66), for $\zeta \rightarrow 0$,

$$
\frac{\overline{\Psi_{N}(1 / \bar{\zeta})}}{X(\zeta)}=-\frac{1}{\zeta}\left(\overline{G_{N}(1 / \bar{\zeta})}+\overline{F_{N}(1 / \bar{\zeta})}\right)=-\frac{1}{\zeta} \overline{F_{N}(1 / \bar{\zeta})}+O(1)
$$

according to (66).

Moreover,

$$
X^{\prime}(\zeta)=\frac{\zeta}{\zeta^{2}+1} X(\zeta)
$$


from which it follows that

$$
\begin{aligned}
\frac{\Psi_{N}^{\prime}(\zeta)}{X(\zeta)} & =\frac{d}{d \zeta}\left(\frac{\Psi_{N}(\zeta)}{X(\zeta)}\right)+\frac{\zeta}{\zeta^{2}+1} \frac{\Psi_{N}(\zeta)}{X(\zeta)}= \\
& =G_{N}^{\prime}(\zeta)+F_{N}^{\prime}(\zeta)+\frac{\zeta}{\zeta^{2}+1}\left(G_{N}(\zeta)+F_{N}(\zeta)\right)
\end{aligned}
$$

Moreover, we have

$$
\frac{\zeta}{\zeta^{2}+1}=\zeta \sum_{j=0}^{\infty}(-1)^{j} \zeta^{2 j}=\zeta \sum_{j=0}^{\infty} \beta_{j} \zeta^{j}
$$

with

$$
\beta_{j}:= \begin{cases}(-1)^{n}, & \text { for } j=2 n, \text { and } n \in \mathbb{N} \cup\{0\} \\ 0, & \text { for } j \text { odd or } j<0\end{cases}
$$

For $|\zeta|<1$, on using (49) in (65), we may approximate $G_{N}(\zeta)$ by means of a Taylor series development around the point $\zeta=0$, according to

$$
G_{N}(\zeta)=\sum_{j=0}^{\infty} g_{j} \zeta^{j}
$$

with $g_{j}$ equal to

$$
g_{j}= \begin{cases}\frac{1}{2}\left(a_{j}-b_{j}\right), & \text { for } 0 \leq j<2 N \\ \frac{1}{2} a_{j}, & \text { for } j \geq 2 N\end{cases}
$$

Substitution of (28) and (69)-(75) into (67) renders the following asymptotic expansion for $\omega_{N}^{\prime}(\zeta) / X(\zeta)$, for $\zeta \rightarrow 0$,

$$
\begin{aligned}
-\frac{\omega_{N}^{\prime}(\zeta)}{X(\zeta)}=\zeta^{-N} & \sum_{i=0}^{N-1}\left(\sum_{j=0}^{i}(j+1) \mu_{j+1} f_{N-1-i+j}\right) \zeta^{i}+ \\
& \zeta^{-N} \sum_{i=0}^{N-1}\left(\sum_{j=0}^{i}(j+1) \mu_{N-i+j}\left(g_{j+1}+f_{j+1}\right)\right) \zeta^{i}+ \\
& \zeta^{-N} \sum_{i=1}^{N-1}\left(\sum_{j=0}^{i-1} \mu_{N+1-i+j} \sum_{l=0}^{j} \beta_{l}\left(g_{j-l}+f_{j-l}\right)\right) \zeta^{i}+O(1),
\end{aligned}
$$

where $f_{N}$ is to be taken equal to zero. The requirement that the right-hand side of (67) must be finite for $\zeta \rightarrow 0$, leads us to the following set of linear equations, from which $f_{k}, 0 \leq k \leq N-1$, can be calculated in terms of $\mu_{k}$ 
and $g_{k}$

$$
\begin{aligned}
& \sum_{j=0}^{i}(j+1)\left(\mu_{j+1} f_{N-1-i+j}+\mu_{N-i+j}\left(g_{j+1}+f_{j+1}\right)\right)+ \\
& \quad \sum_{j=0}^{i-1} \mu_{N+1-i+j} \sum_{l=0}^{j} \beta_{l}\left(g_{j-l}+f_{j-l}\right)=0, \quad \text { for } 0 \leq i \leq N-1,
\end{aligned}
$$

where $f_{N}=0$. So far, we have derived explicit expressions for $a_{k}$ and $b_{k}$ in terms of $\mu_{k}$. Also, we have found an explicit expression for $G_{N}(\zeta)$ in terms of $a_{k}$ and $b_{k}$. By substituting this solution into the holomorphy condition given by (36), we found a set of linear equations for $f_{k}$ in terms of $\mu_{k}$ and $g_{k}$. By solving this set of equations and substituting the solution into (50), we obtain an expression for $\Psi_{N}(\zeta)$, from which we can calculate the complex velocity $w_{N}$.

For $\zeta \in G_{\zeta}^{+}$, we have the following relation for the total (i.e. inclusive the Poiseuille part; see (16)) complex velocity

$$
w_{N}(\zeta)=\frac{1}{2}-\frac{3}{2} \operatorname{Im}\left(m_{N}(\zeta)\right)^{2}+w_{N 1}(\zeta)
$$

where $w_{N 1}(\zeta)$ is given by (30). Substitution of the first equation of (35) and (36) into (30) renders the following expression for $w_{N 1}$, for $\zeta \in G_{\zeta}^{+}$,

$$
w_{N 1}(\zeta)=\Psi_{N}(1 / \bar{\zeta})-\Psi_{N}(\zeta)+\frac{m_{N}(\zeta)-m_{N}(1 / \bar{\zeta})}{\overline{m_{N}^{\prime}(\zeta)}} \overline{\Psi_{N}^{\prime}(\zeta)}
$$

where $\Psi_{N}(\zeta)$ is given by (41).

At this point, we have obtained with (79) a possibility to calculate explicitly the velocity in any point in the flow front area, where a fountain flow is observed. In the next section, this will be made explicit with numerical results for the velocity components for the approximated case: $m_{N}(\zeta)=\zeta$, that is in case the flow front is approximated by a semi-circle.

For $\xi \in \Gamma_{f}^{\prime}$, we need to take the limit for $\zeta \rightarrow \xi, \zeta \in G_{\zeta}^{+}$, which renders the following equation for $w_{N 1}$ for $\xi \in \Gamma_{f}^{\prime}$,

$$
\begin{aligned}
w_{N 1}(\xi) & =\Psi_{N}^{-}(\xi)-\Psi_{N}(\xi)^{+}= \\
& =X^{+}(\xi)\left(\sum_{k=-2 N}^{-1} a_{k} \xi^{k}+\sum_{k=0}^{2 N-1} b_{k} \xi^{k}-2 \sum_{k=0}^{N-1} f_{k} \xi^{k}\right) .
\end{aligned}
$$

\subsection{Results for circular flow front approximation}

In this section, we will calculate explicit numerical values for the velocity inside the flow area, close to the flow front region. However, we will do this 
only within an approximation, in which we approximate the flow front by a semi-circle, meaning that we use $z=m(\zeta)=\zeta$. Although results of [3] show that $\mu_{1} \approx 1$ and dominant over over the other coefficients $\mu_{k}$ (see for instance (51)), this is certainly not exactly true. However, as we are especially interested in the flow behaviour close to the flow front, where this approximation is best, this seems to us an acceptable approach. We could just as well have used the, more exact, results for $N=3$ as given in (51), and in fact for a part we did so, but not only were the calculations more complex then, the relevant results were nearly the same (differences less then a few procent).

Therefore, we stick to this approximation, for which we have $N=1$ and $\mu_{0}=0$ and $\mu_{1}=1$. This implies that in (50), we only need to know the coefficients $a_{-2}, a_{-1}, b_{0}, b_{1}$, and $f_{0}$. From (57), (58), and (77), we obtain

$$
a_{-2}=b_{1}=2 f_{0}=\frac{3}{8}, \quad a_{-1}=b_{0}=0
$$

For $g(\zeta)$ (we omit the index $N(=1)$ from now on) we obtain from (47)

$$
g(\zeta)=\frac{3}{8}\left(\zeta^{2}+2-\zeta^{-2}\right)
$$

and all this leads us with (50) to the following expression for $\Psi(\zeta)$

$$
\Psi(\zeta)=\frac{3}{16}\left[\zeta^{2}+2-\zeta^{-2} \pm \sqrt{1+\zeta^{2}}\left(\zeta^{-2}-1+\zeta\right)\right], \quad \zeta \in \mathbb{C} \backslash \Gamma_{f}^{\prime},
$$

where the $+\operatorname{sign}$ holds for $\zeta \in G_{\zeta}^{+}$and the $-\operatorname{sign}$ for $\zeta \in G_{\zeta}^{-}$.

This means that for $\zeta \in G_{\zeta}^{+}$, when $1 / \bar{\zeta} \in G_{\zeta}^{-}$,

$$
\Psi(1 / \bar{\zeta})=\frac{3}{16}\left[\bar{\zeta}^{-2}+2-\bar{\zeta}^{2}-\sqrt{1+\bar{\zeta}^{2}}\left(\bar{\zeta}-\bar{\zeta}^{-1}+\bar{\zeta}^{-2}\right)\right]
$$

From (78) and (79) and with $m(\zeta)=\zeta=z$, the following expression for the total complex velocity $w(\zeta)=w(z)$ in the flow front region, $z \in G_{\zeta}^{+}$, follows

$$
w(z)=\frac{1}{2}+\frac{3}{8}(z-\bar{z})^{2}-\Psi(z)+\Psi(1 / \bar{z})+(z-1 / \bar{z}) \overline{\Psi^{\prime}(z)}
$$

The use of (83) and (84) in (85) results in an explicit, completely analytical, expression for $w(z)$ in terms of $z=x+\mathrm{i} y$ and $\bar{z}$. This expression will be used in the next section to calculate the velocity field of the fountain flow in the flow front region. 


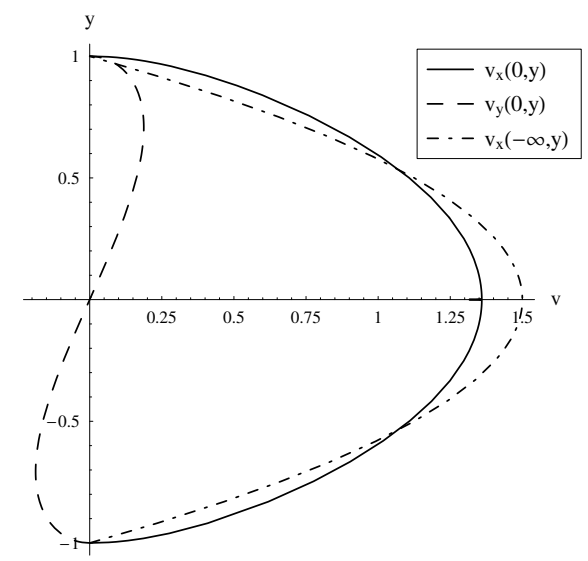

a) Velocities

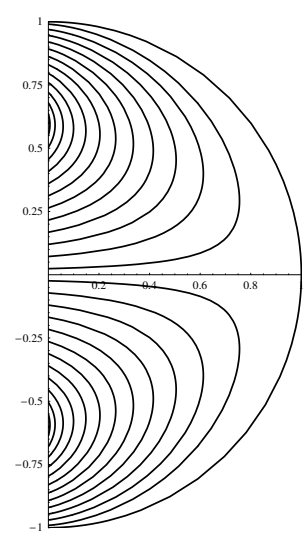

b) Streamlines

Fig. 5. a) The velocity components at the border $x=0$ of the flow front region (axial velocity $v_{x}$ : full line; transverse velocity $v_{y}$ : dashed line) compared to the axial velocity in Poiseuille flow (dot-dashed line); b) Streamlines in co-moving frame.

\section{Velocity field, deformation and rotation, and stresses behind the flow front}

The results of Section 4 will be used here to calculate the velocity field in and behind the flow front region. The thus calculated velocity is an approximation of the actual velocity in the fluid, only accurate in the flow front region $(\operatorname{Re}(z)>0)$. Behind the flow front region, we assume the flow to be a Poiseuille flow. In Figure 5a), the velocity profiles at the border of the flow front region, i.e. at $\operatorname{Re}(z)=0$, according to (85) are compared to a fully developed Poiseuille flow. This figure shows that the differences there are already small (less than 10\%). So, the approach to approximate the flow directly behind the flow front region by a Poiseuille flow seems justified.

Typical streamline patterns are depicted in Figure 5b), showing the streamlines relative to the flow front; this figure clearly demonstrates the fountain flow effect. Comparing these results with results found by numerical means in e.g. [1], [2, Fig. 8], [9], and [10, Fig. 4], a practically complete correspondence is found. In [2], see Figs. 13-14, the numerically simulated shape of the flow front shows a close resemblance to a semi-circle, in correspondence to our Figure 3. This motivated our choice made in Section 4.2 to approximate the flow front by a semi-circle. Moreover, it is noticeable that no qualitative differences are found in [2] between the numerical simulations for Newtonian and for shear-thinning fluids.

In analogy with [9] and [6], we have analysed the behaviour (deformation, rotation) of material, line or surface, elements in injection flow. In Figure 6 


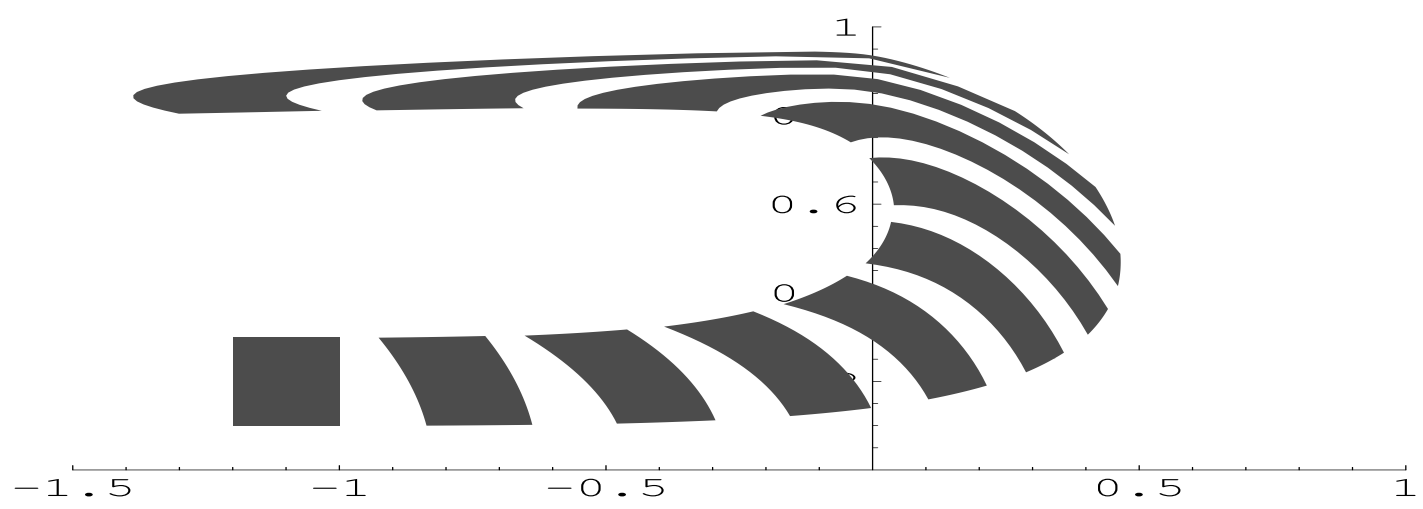

Fig. 6. Behaviour in time of an initially rectangular area element when passing through the flow front region, relative to the flow front

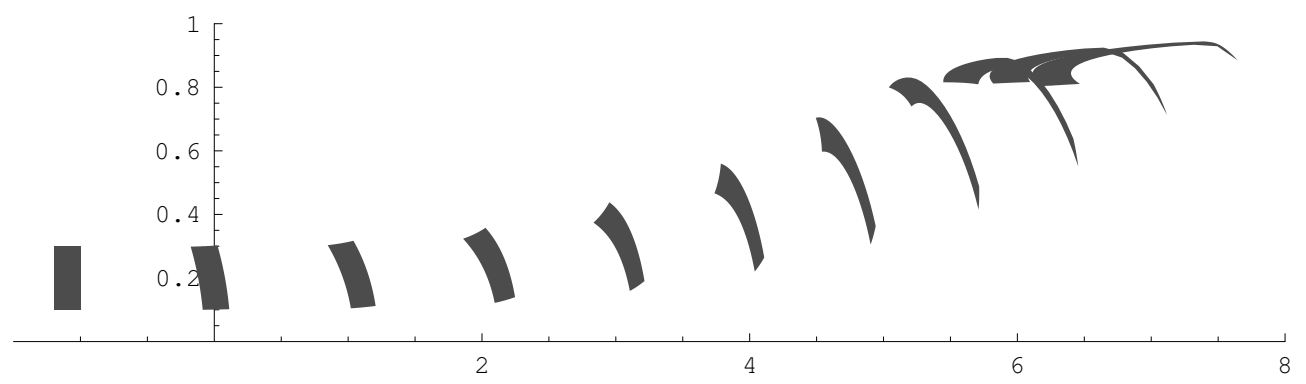

Fig. 7. Behaviour in time of an initially rectangular area element when passing through the flow front region, with respect to the inertial coordinate system

and Figure 7, we show the behaviour of a rectangular material element entering the fountain flow region. In the first figure, the behaviour relative to the flow front (i.e. in a co-moving frame) is illustrated, while the same behaviour, but now in an inertial frame, is presented in the second figure. We observe how the element is stretched, spills over when reaching the flow front, and becomes very strongly stretched at the wall. Further we notice in Figure 7 that the tail of the stretched element remains normal to the wall up to the very last moment of reaching the fixed wall. Following [6], we also looked at the deformation of an initially straight transverse line; the result is depicted in Figure 8. The latter figure shows striking alikeness with Fig. 5 in [6], obtained from finite elements calculations for a Carreau fluid. Watch especially the typical V-shapes near the wall in the last picture. Finally, we show the behaviour of a material element consisting of two material line elements, initially forming a right angle in Figure 9 . We see that the right angle is folded to a small sharp angle. Moreover, following the element initially orientated in the axial direction, we once more notice that this line element is orientated almost normal to the wall when approaching this wall, and only when it is very near to the wall it suddenly flips over to an orientation parallel to the wall. In practice, the polymer melt solidifies at the cooled walls immediately after injection, forming solid (glass) layers at the walls. This means that the polymer chains that are initially in axial direction, will be frozen in having 

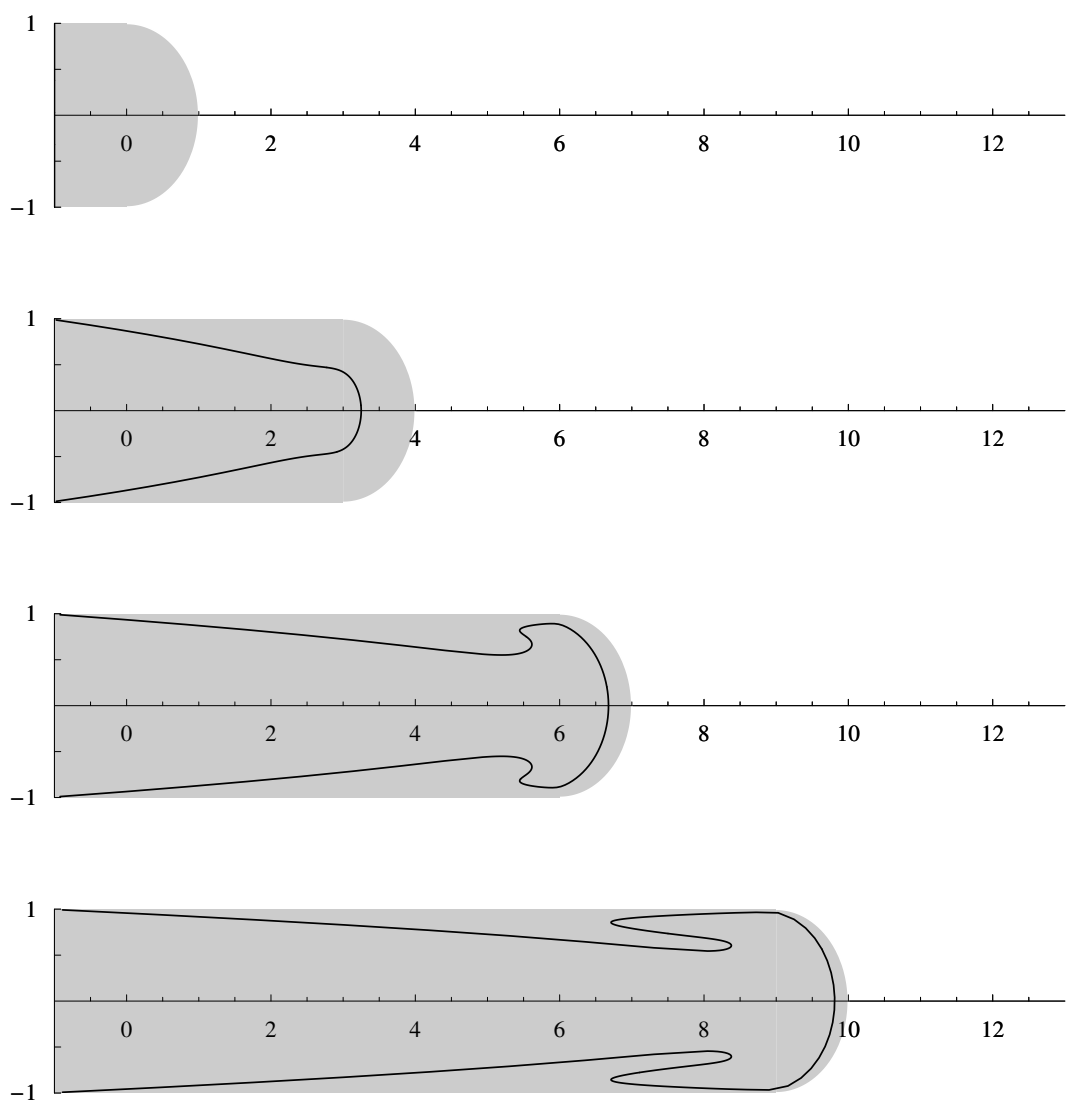

Fig. 8. Deformation of an initially straight transverse line element at four increasing times.

an orientation perpendicular to the walls. This has an essential effect on the frozen-in stresses, and then also on the birefringence, near the walls. This effect is confirmed by numerical simulations and experimental observations, see e.g. [13, Sect. 5.1]. The stresses in the fluid, especially in the flow front region, can be calculated from (25). For the stresses, we are interested in the following aspects:

(1) The pressure $p$, given by

$$
\begin{aligned}
p & =-\frac{1}{2}\left(T_{x x}+T_{y y}\right)=-\frac{1}{2} N(z, \bar{z}) \\
& =\Omega_{1}^{\prime}(z)+\overline{\Omega_{1}^{\prime}(z)}-\frac{3}{4}(z+\bar{z})=p(x, y) .
\end{aligned}
$$

We have plotted $p$ along the upper wall $(y=1, x<0)$ and the upper part 


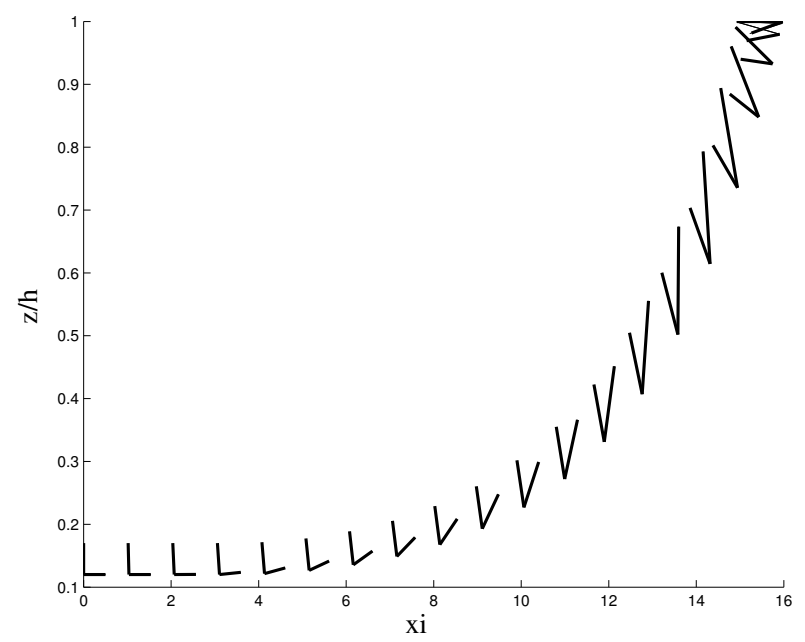

Fig. 9. Transformation of a material element consisting of two line elements, initially forming a right angle

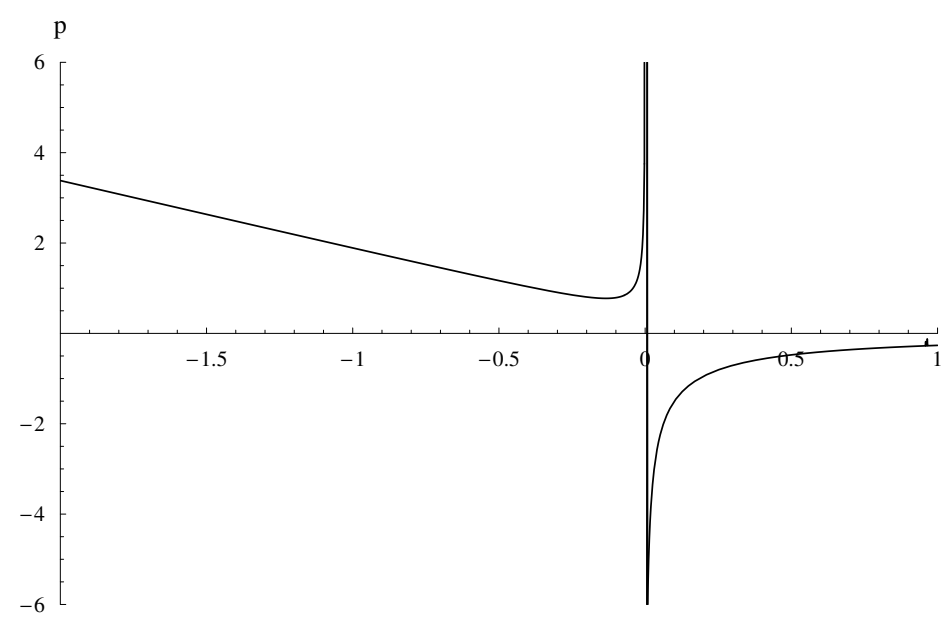

Fig. 10. Pressure along the upper wall and the upper part of the flow front

of the flow front $\Gamma_{f}\left(x^{2}+y^{2}=1, x>0, y>0\right)$ in Figure 10. This graph shows a singularity in the point $(x, y)=(0,1)$. Our analysis predicts that this singularity has an order of $-1 / 2$ (a square root singularity); this can be inferred from the behaviour of $\Psi^{\prime}$, and more specifically from that of the Plemelj function in it, for $z \rightarrow \pm \mathrm{i}$. The result found here is in good correspondence with that found in [2, Fig.11] for shear-thinning fluids.

(2) The first normal stress difference $N_{1}$, can be found from

$$
\begin{aligned}
N_{1} & =T_{x x}-T_{y y}=\operatorname{Re} S(z, \bar{z}) \\
& =z \overline{\Omega_{1}^{\prime \prime}(z)}+\bar{z} \Omega_{1}^{\prime \prime}(z)+\overline{\omega_{1}^{\prime \prime}(z)}+\omega_{1}^{\prime \prime}(z)=N_{1}(x, y) .
\end{aligned}
$$

However, we did not depict the results, because we do not expect that our Newtonian model will give a realistic picture of this normal stress difference due to the strong stretches observed in the flow front region. 


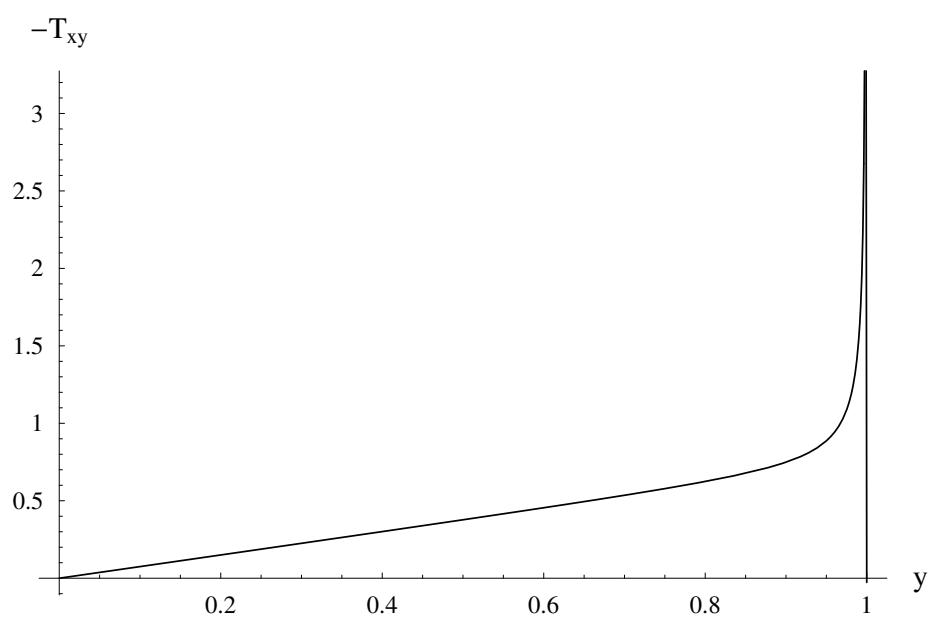

Fig. 11. Shear stress $T_{x y}$ along the front line $x=0$

These large elongations can only rightly be incorporated by a nonlinear viscoelastic model, that accounts for the highly elastic influence on the (normal) stresses caused by these elongations.

(3) The shear stress $T_{x y}$, given by

$$
\begin{aligned}
T_{x y} & =\frac{1}{2} \operatorname{Im} S(z, \bar{z}) \\
& =\frac{1}{2}\left(z \overline{\Omega_{1}^{\prime \prime}(z)}-\bar{z} \Omega_{1}^{\prime \prime}(z)+\overline{\omega_{1}^{\prime \prime}(z)}-\omega_{1}^{\prime \prime}(z)-\frac{3}{4 \mathrm{i}}(z-\bar{z})\right) .
\end{aligned}
$$

In Figure 11, the shear stress along the border $x=0$ of the flow front region is plotted as a function of $y$ for $0<y<1$. We see almost linear behaviour on the main part of this line; only in the end point near $y=1$ a very steep behaviour representing the square root singularity in the stress at the separation point $x=0, y=1$ is observed. This behaviour is characteristic for Newtonian fluids, when a no-slip condition is maintained (compare for instance with [11, Fig.5]).

(4) The second invariant $I_{T}$, can be found from

$$
I_{T}=\frac{1}{2}(\operatorname{tr} \mathcal{T})^{2}-\frac{1}{2} \operatorname{tr} \mathcal{T}^{2}=2 p^{2}-\frac{1}{2} \operatorname{tr} \mathcal{T}^{2}
$$

With

$$
\operatorname{tr} \mathcal{T}^{2}=T_{x x}^{2}+T_{y y}^{2}+2 T_{x y}^{2}=4 p^{2}-2\left(T_{x x} T_{y y}-T_{x y}^{2}\right),
$$

it follows that

$$
I_{T}=T_{x x} T_{y y}-T_{x y}^{2}=p^{2}-\frac{1}{4} S(z, \bar{z}) \overline{S(z, \bar{z})},
$$

as can be deduced from (25).

The second invariant of the stress tensor, $I I_{T}$, is of practical importance, 
because it is directly related to birefringence, see [13], as can occur in injection moulded compact discs. We did not plot our results for $I_{T}$ for the same reason as mentioned for the normal stress difference. However, there seems to be an essential difference in the behaviour in $I_{T}$, especially very close to the walls, found from numerical simulations in which the shape of the flow front is taken into account compared to similar ones in which the flow front is replaced by a straight line (and in which the thin-layer approximation is applied over the whole flow region); see [13]. Moreover, although birefringence is due to the elongations built up in the fountain flow phase, the stresses causing this birefringence are frozen-in in a later stage after the mould is completely filled. As this stage is not considered in this work, we refrain from further considerations on $I_{T}$.

\section{Conclusions}

A complete analytical model has been developed for the injection moulding of a Newtonian fluid between two parallel plates. Explicit formulas have been derived for the shape of the free flow front (a free-boundary problem) and for the description of the fountain flow in the flow front region. These analytical results were derived by using complex function theory, inclusive a conformal mapping of the flow front onto a circle. The problem was reformulated in terms of a Hilbert problem, that could be solved by means of Plemelj functions. These Plemelj functions are characteristic for the singularities in the separation point, where the fluid separates from the fixed wall. A square root singularity in the stresses is found there. For a global characterization of the flow, the approximation of the actual flow front by a semi-circle is appropriate; this seems to hold irrespective of the rheology of the fluid. It was found that the velocity distribution at the border of the flow front region was very close to that of the Poiseuille flow. This supports the expectation that the flow will be a fully developed Poiseuille flow already a few times ( 2 or 3 ) the thickness $h$ behind the flow front. For the region behind the flow front, the full formula for $m(\zeta)$ according to (28) and (51) must be used; thus obtained results (not published in this paper) confirm the expectation mentioned above. We have performed a few calculations using the full formula for $m(\zeta)$, e.g. for calculating the flow patterns within the flow front region; only minor differences with the results published here for $m(\zeta)=\zeta$ are found .

Comparison with existing literature reveals that our theoretical/analytical results show on the whole very good correspondence with numerical and experimental results reported in literature, both qualitative and, whenever possible, quantitative. Kinematical results, such as flow-front shape, velocities, and deformations and rotations (orientation), seem to be quite insensitive to the rheology of the fluid. Of course, this does not hold for the stresses, especially 
for the normal stress differences, which are very sensitive to elastic effects. These elastic effects become dominant whenever large elongations occur, as in the fountain flow in the flow front region. Moreover, this insensitivity is only present for regular flows; in instability investigations, see [17] or [18], the specific rheology becomes crucial. Nevertheless, for the unperturbed flow, the rheology is far less relevant.

As our results are purely analytical, they can be incorporated in further research to typical aspects of of the free flow front motion in injection moulding. To mention two examples, the flow patterns found here can be used:

- As input for the convection-diffusion temperature problem that is related to the injection moulding of a hot polymer melt into a cold mould. This problem, and specifically the thermal boundaries that show up in it, will be the subject of the forthcoming paper [20]; see also [21].

- As an unperturbed solution for stability problems investigating the (in)stability of slightly perturbed flow front motions ("wobbling of the flow front"). When incorporating also thermal effects by means of [20], the influence of temperature on e.g. viscosity, and after that the influence of all this on the free motion of the flow front, the effect of an initial asymmetric perturbation on the motion of the front can be analysed; see [21] or [22]. The instability of the flow front motion is an important problem in the practice of injection moulding, as it causes surface defects on the final product; see [16], [17] and $[18]$.

\section{References}

[1] C. Diereck, Stream function formulation of Stokes problems with stress boundary conditions, Report R479 (Philips Research Laboratory, Brussels, 1984).

[2] H. Mavridis, A.N. Hrymak, J. Vlachopolous, Finite element simulation of fountain flow in injection moulding, Polym. Eng. Sci. 26 (1986) 449-454.

[3] J.C.W.van Vroonhoven, W.J.J. Kuijpers, A free boundary problem for viscous fluid flow in injection moulding, J. Eng. Math. 24 (1990) 151-165.

[4] B. Friedrichs, S.I. Güçeri, A novel hybrid numerical technique to model 3-D fountain flow in injection moulding process, J. Non-Newtonian Fluid Mech. 49 (1993) 141-173.

[5] A. Almeida, L. Faria, R. Febra, Refined two-dimensional models for the analysis of free boundary flows between parallel plates, Comput. Methods Appl. Mech. Engrg. 151 (1998) 163-180.

[6] T. Nguyen-Chung, G. Mennig, Non-isothermal transient flow and Molecular orientation during injection mold filling, Rheol. Acta 40 (2001) 67-73. 
[7] E. Pichelin, T. Coupez, Finite element solution of the 3D mold filling problem for viscous incompressible fluid, Comput. Methods Appl. Mech. Engrg. 163 (1998) 359-371.

[8] H. Yokoi, N. Masuda, H. Mitsuhata, Visualisation analysis of flow front behavior during filling process of injection mold cavity by two-axis tracking system, J. Mat. Proc. Techn. 130-131 (2002) 328-333.

[9] H. Mavridis, A.N. Hrymak, J. Vlachopolous, The effect of fountain flow on molecular orientation in injection moulding, Journal of Rheol. 32 (1988) 639663.

[10] T. Sato, S.M. Richardson, Numerical simulation of fountain flow problem for viscoelastic liquids. Polymer Engineering Science 35 (1995) 805-812.

[11] M.R. Kamal, S.K. Goyal, E. Chu, Simulation of injection mold filling of viscoelastic polymer with fountain flow. AIChE Journal 34 (1988) 94-106.

[12] Z. Tadmor, Molecular orientation in injection moulding, J. Appl. Polym. Sci. 18 (1974) 1753-1772.

[13] R. Wimberger-Friedl, Orientation, Stress and Density Distributions in Injection-Moulded Amorphous Polymers Determined by Optical Techniques, Ph.D.Thesis, Eindhoven University of Technology (Eindhoven, The Netherlands, 1991).

[14] C.F. Hung, Y.K. Shen, Numerical simulation of fiber orientation in injection mold filling, Int. Comm. Heat Mass Transfer 22 (1995) 791-802.

[15] G. Vincent, E. Devilers, J.F. Agassant, Fibre orientation calculation in injection moulding of reinforced thermoplastics, J. Non-Newtonian Fluid Mech. 73 (1997) 317-326.

[16] A.M. Grillet, A.C.B. Bogaerds, G.W.M. Peters, F.P.T. Baaijens, M. Bulters, Numerical analysis of flow mark surface defects in injection molding flow, Journal of Rheol. 46 (2002) 651-669.

[17] A.C.B. Bogaerds, Stability analysis of viscoelastic flows, Ph.D.Thesis, Eindhoven University of Technology (Eindhoven, The Netherlands, 2002).

[18] A.C.B Bogaerds, M.A. Hulsen, G.W.M. Peters, F.P.T. Baaijens, Stability analysis of injection molding flows. Journal of Rheology, submitted

[19] A.H. England, Complex Variable Methods in Elasticity (Wiley-Interscience, London, 1971).

[20] H.J.J. Gramberg, A.A.F. van de Ven, Temperature distribution in a Newtonian fluid injected between two semi-infinite plates, forthcoming.

[21] H.J.J. Gramberg, A.A.F. van de Ven, Thermally induced flow front instabilities in injection moulding, in: A.M. Anile, V. Capasso, A. Greco eds., Progress in Industrial Mathematics at ECMI 2000 (Springer, Berlin, 2002). 
[22] A.A.F. van de Ven, Modelling of industrial processes for polymer melts: Extrusion and injection moulding, in: V. Capasso ed., Mathematical Modelling for Polymer Processing. Polymerization, Cristallization, Manufacture (Springer, Berlin, 2002) 263-309. 